\title{
Tenascin expression in endomyocardial biopsy specimens in patients with dilated cardiomyopathy: distribution along margin of fibrotic lesions
}

Akiko Tamura, Shozo Kusachi, Kunio Nogami, Asami Yamanishi, Yutaka Kajikawa, Satoshi Hirohata, Takao Tsuji

\begin{abstract}
Objective-To examine the hypothesis that tenascin, an extracellular matrix glycoprotein, contributes to fibrotic changes in dilated cardiomyopathy.

Methods-The localisation of tenascin in biopsy specimens of the hearts obtained from eight patients with dilated cardiomyopathy was examined using staining by the avidin-biotin-peroxidase complex method

Results-(1) Perimysium and endomysium. Although positive staining for tenascin was observed in the enlarged perimysium and endomysium in all patients, moderately intense staining was characteristically observed near the replacement fibrotic lesions. In the narrow perimysium and endomysium of the myocardium not containing replacement fibrotic lesions, tenascin was not present, as in the control specimens. (2) Replacement fibrotic lesions. Non-homogeneous positive staining for tenascin was detected in all replacement fibrotic lesions examined. Intense tenascin deposition was observed in the peripheral portion of the replacement fibrotic lesions. The tenascin staining observed in the small replacement fibrotic lesions was more intense than that in the large lesions.

Conclusions-Tenascin contributes to the development of the fibrotic changes seen in the dilated cardiomyopathic heart. Its characteristic location, specifically the distribution along the margin of the fibrosis, suggests that fibrotic change is a continuous process in hearts with dilated cardiomyopathy.
\end{abstract}

The First Department Okayama University

Medical School,

Okayama, Japan

A Tamura

S Kusachi

K Nogami

A Yamanishi

Y Kajikawa

S Hirohata

T Tsuji

Correspondence to:

Shozo Kusachi MD, The 1st Dept of Internal Medicine, Okayama University Medica chool, 2-5-1, Shikata-cho, Okayama 700, Japan

Accepted for publication

8 August 1995
(Heart 1996;75:291-294)

Keywords: glycoprotein; extracellular matrix components; polyclonal antibody, cardiac fibrosis. der of unknown aetiology characterised by left ventricular cavity enlargement and wall thinning, in association with reduced left ventricular wall motion.

The major histopathological characteristics sis-a critical feature - and myocyte degeneration. ${ }^{1}$ The extracellular matrix (ECM), the
Idiopathic dilated cardiomyopathy is a disorof dilated cardiomyopathy are cardiac fibro- major components of which are collagens, proteoglycans, and glycoproteins, plays a vital role in cardiac fibrotic changes.

Tenascin is an ECM glycoprotein which was first detected by Chiquet and Fambrough. ${ }^{2}$ The expression of tenascin, unlike that of other ECM components, is spatially and chronologically restricted. Tenascin is found in the migratory pathways during development, and its appearance in the ECM in migratory pathways correlates with the initiation of cell migration. ${ }^{3}$ In addition, tenascin appears at sites of tissue repair ${ }^{4-6}$; both the repair process and its development involve many molecular mechanisms. The characteristic distribution and expression of tenascin thus suggests that it may have a certain role in tissue repair, such as fibrosis following myocyte degeneration. However, its characteristics in the dilated cardiomyopathic heart have not been elucidated.

Employing the avidin-biotin-peroxidase complex (ABC) method, ${ }^{7}$ we therefore investigated the localisation of tenascin in endomyocardial biopsy specimens obtained from hearts with dilated cardiomyopathy.

\section{Methods}

PATIENTS AND MATERIAL

We performed endomyocardial biopsy in eight patients with dilated cardiomyopathy after obtaining their informed consent. Dilated cardiomyopathy was diagnosed according to the diagnostic criteria of WHO/ISFC. ${ }^{8}$ The clinical characteristics of the patients studied are summarised in table 1 . Samples were obtained from the lateral wall of the left ventricle. Three to five biopsy specimens were obtained in each patient; two or three specimens stained with haematoxylin-eosin and Azan-Mallory served for conventional light microscope examination and the remaining specimens were subjected to immunohistochemical study.

Since it was impossible to obtain normal heart biopsy specimens, left ventricular specimens obtained from two necropsy cases without any heart disease served as controls.

IMMUNOHISTOCHEMISTRY

For the immunohistochemical study, we stained the frozen biopsy specimens by the avidin-biotin-peroxidase complex (ABC) method. ${ }^{7}$ Briefly, the primary antibody used in this study was rabbit anti-human tenascin 
Table 1 Patient characteristics

\begin{tabular}{|c|c|c|c|c|c|c|c|}
\hline \multirow[b]{2}{*}{ Patient no } & \multirow[b]{2}{*}{ Age (y) } & \multirow[b]{2}{*}{ Sex } & \multirow[b]{2}{*}{ Symptoms } & \multirow[b]{2}{*}{$E C G$} & \multicolumn{2}{|l|}{ Echo } & \multirow{2}{*}{$\begin{array}{l}L V G \\
E F(\%)\end{array}$} \\
\hline & & & & & $\operatorname{LVDd}(\mathrm{mm})$ & $E F(\%)$ & \\
\hline $\begin{array}{l}1 \\
2 \\
3 \\
4 \\
5 \\
6 \\
7 \\
7 \\
8\end{array}$ & $\begin{array}{l}68 \\
24 \\
49 \\
69 \\
48 \\
56 \\
45 \\
49\end{array}$ & $\begin{array}{l}M \\
M \\
F \\
F \\
M \\
M \\
M \\
M\end{array}$ & $\begin{array}{l}\text { Palpitation } \\
\text { Exertional dyspnoea } \\
\text { General fatigue } \\
\text { Chest discomfort } \\
\text { Exertional dyspnoea } \\
\text { Chest discomfort } \\
\text { Exertional dyspnoea } \\
\text { Orthopnoea }\end{array}$ & $\begin{array}{l}\text { Ventricular premature beats } \\
\text { Poor } R \text { wave progression } \\
\text { Inverted T wave in V3-V6 } \\
\text { Poor } R \text { wave in V1-4 } \\
\text { Right axis deviation } \\
\text { Ventricular premature beats } \\
\text { Sinus bradycardia } \\
\text { Inverted T wave in V4-V6 }\end{array}$ & $\begin{array}{l}60 \\
73 \\
60 \\
58 \\
68 \\
63 \\
59 \\
67\end{array}$ & $\begin{array}{l}29 \\
26 \\
50 \\
30 \\
28 \\
37 \\
46 \\
27\end{array}$ & $\begin{array}{l}27 \\
26 \\
43 \\
47 \\
32 \\
41 \\
44 \\
23\end{array}$ \\
\hline
\end{tabular}

M, male; F, female; echo, echocardiography; LVG, left ventriculography; LVDd, left ventricular end diastolic dimension; $\mathrm{EF}$, ejection fraction.

polyclonal antibody (Chemicon) diluted 1:500 $(2 \mu 1 / \mathrm{ml})$ with phosphate buffered saline (PBS) containing $1.5 \%$ normal goat serum. The sections of the specimens were incubated with the diluted primary antibody for $10 \mathrm{~h}$ at $4^{\circ} \mathrm{C}$. The second antibody used in this study was goat anti-rabbit IgG serum (Funakoshi) diluted 1:1000 with PBS containing 1.5\% normal goat serum. The sections were incubated with the second antibody for $2 \mathrm{~h}$ at room temperature, reacted with avidin-biotinperoxidase complex, and finally coloured with diaminobenzidine. For cell nucleus staining, the sections were incubated with $0.1 \mathrm{M}$ veronal acetate buffered $1 \%$ methyl green for $10 \mathrm{~min}$.

\section{IMMUNOHISTOCHEMICAL CONTROL}

PROCEDURE

The following procedures were performed: negative control staining with normal rabbit serum instead of primary rabbit antibody for each staining; and an immunoabsorption test using purified human tenascin antigen (Nippon Koutai Kenkyusho) to the antibody.

\section{ANALYSIS}

Since the extracellular matrix components of the heart are located in the perimysium and endomysium and in lesions where fibrosis has replaced myocyte necrosis (replacement fibrotic lesions), we analysed the localisation

\section{Table 2 Conventional light microscopic findings}

\begin{tabular}{lllll}
\hline Patient & $\begin{array}{l}\text { Myocyte } \\
\text { degeneration }\end{array}$ & $\begin{array}{l}\text { Myocyte } \\
\text { hypertrophy }\end{array}$ & $\begin{array}{l}\text { Intercellular } \\
\text { fibrosis }\end{array}$ & $\begin{array}{l}\text { Replacement } \\
\text { fibrosis }\end{array}$ \\
\hline 1 & + & + & ++ & ++ \\
2 & ++ & + & ++ & +++ \\
3 & ++ & +++ & ++ & + \\
4 & + & + & + & + \\
5 & + & + & ++ & + \\
6 & ++ & ++ & ++ & + \\
7 & ++ & + & ++ & +++ \\
8 & ++ & + & ++ & +++ \\
\hline
\end{tabular}

Table 3 Immunohistochemical staining results

\begin{tabular}{llllll}
\hline & \multicolumn{2}{l}{ Intercellular spaces } & & & \multicolumn{2}{l}{ Replacement fibrotic lesions } \\
\cline { 2 - 3 } \cline { 5 - 6 } Patient & Dilated & Non-dilated & & Small & Large \\
\hline 1 & - & - & ++ & + \\
2 & +++ & + & ++ & + \\
3 & ++ & + & + & + \\
4 & ++ & - & ++ & + \\
5 & + & - & ++ & + \\
6 & ++ & + & +++ & + \\
7 & ++ & & & & ++ \\
8 & & & & &
\end{tabular}

of tenascin in these regions. The extent of staining was graded as follows: -, no staining; + , slight but apparent; ++ , moderate; and +++ , strong. Inter- and intraobserver differences were evaluated using sample photographs. All assessments were conducted by two investigators in blinded manner. When the two investigators disagreed, an additional blinded observer assessed the samples.

\section{Results}

CONVENTIONAL LIGHT MICROSCOPIC FINDINGS The conventional light microscopic findings in each of the eight patients studied are summarised in table 2. Considerable fibrosis associated with myocyte necrosis, consistent with the characteristics of the heart with dilated cardiomyopathy, was observed in all eight patients.

\section{IMMUNOHISTOCHEMICAL STAINING RESULTS} Immunohistochemical control procedure

No positive staining was observed in negative control staining with normal rabbit serum instead of primary antibody. Positive staining in the specimen from the hearts with dilated cardiomyopathy disappeared after immunoabsorption. The specificity of the staining results was confirmed by these results. No positive staining was observed in the control specimen. The results of immunohistochemical staining for tenascin are summarised in table 3.

\section{Perimysium and endomysium}

Positive staining for tenascin was characteristically observed in the perimysium and endomysium (intercellular spaces) around the replacement fibrotic lesions. The staining for tenascin was detected in enlarged perimysium and endomysium in all patients except patient 1 , and intense staining (grade ++ or +++ ) for tenascin was observed in the enlarged endomysium near the replacement fibrotic lesions (fig 1A). Similarly, in some specimens (from patients 2 and 4 ) the positive staining was observed in the non-enlarged endomysium, and these tenascin positive lesions were also located near the replacement fibrotic lesions. The staining intensity in these lesions varied from lesion to lesion.

On the other hand, in the narrow perimysium and endomysium of the myocardium not containing the replacement fibrotic lesions, antibody results for tenascin confirmed that tenascin was not present, as in the control specimens. 

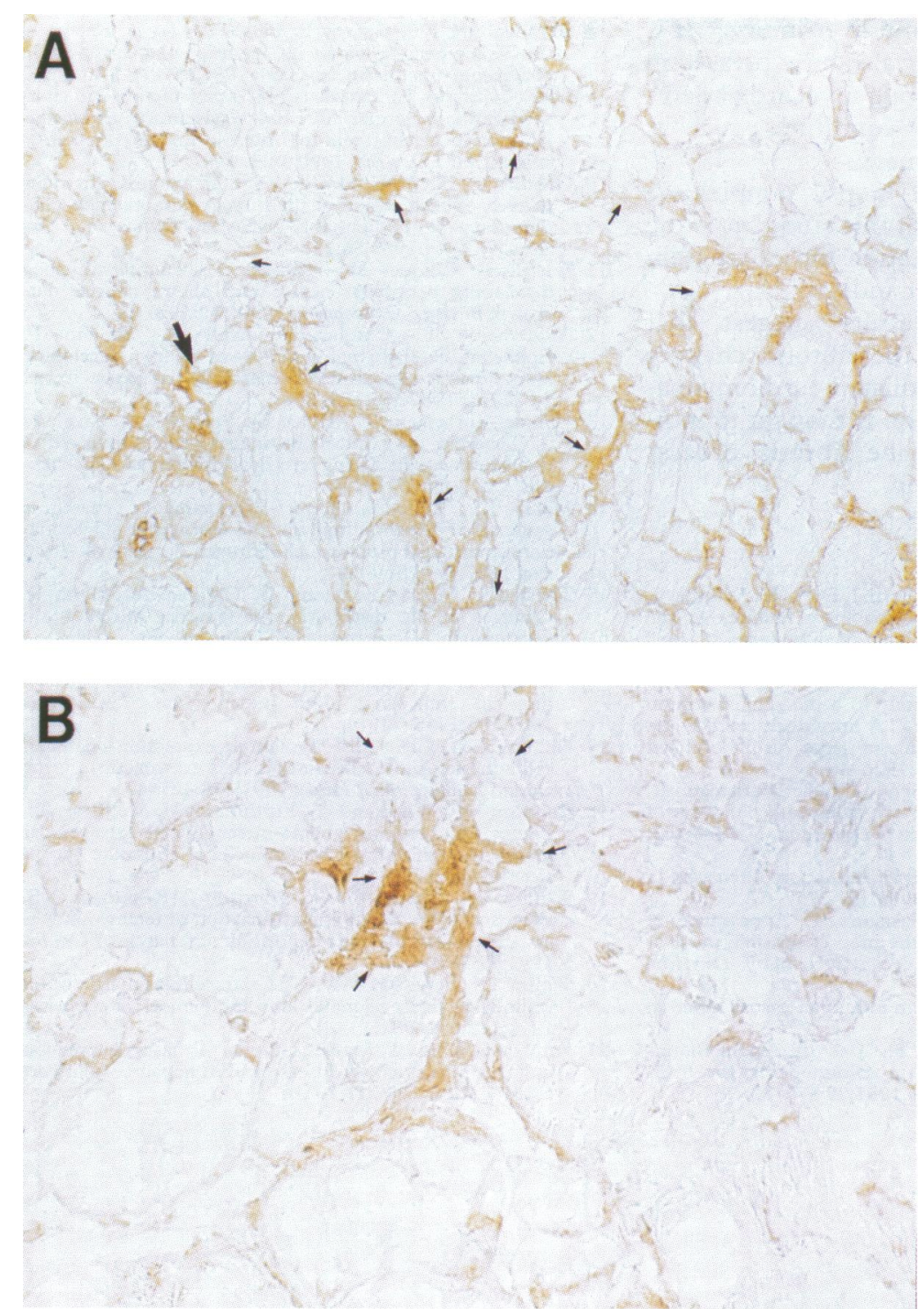

(A) Tenascin staining in a larger replacement fibrotic lesion $(\times 194)$. Positive staining is distributed in the peripheral zone of the replacement fibrotic lesion. Intense staining (grade +++ ) is observed in the enlarged endomysium near the replacement fibrotic lesions (an example of such staining is indicated by the thick arrow). Tenascin deposition (++or $+++)$ was observed in the peripheral portion of the replacement fibrotic lesions. Thin arrows indicate the edge of the replacement fibrotic lesion. (B) Tenascin staining in a smaller replacement fibrotic lesion $(\times 387)$. Intense staining (grade +++ ) for tenascin is seen. The tenascin staining in this replacement fibrotic lesion is more intense than that shown in (A). Arrows indicate the edge of the replacement fibrotic lesion.

\section{Replacement fibrotic lesions}

In all replacement fibrotic lesions, non-homogeneous positive staining for tenascin was detected (fig $1 \mathrm{~A}$ and $1 \mathrm{~B}$ ). The staining intensity in the replacement fibrotic lesions also varied from lesion to lesion. Intense tenascin deposition $(++$ or +++$)$ was observed in the peripheral portion of the replacement fibrotic lesions (fig 1A), but was not observed in the central portion of the replacement fibrotic lesions. The tenascin staining seen in the small replacement fibrotic lesions was more intense than that in the large lesions (fig 1B).

\section{Discussion}

The findings indicate that tenascin is generally distributed in the enlarged perimysium and endomysium near the replacement fibrotic lesions close to necrotising myocytes and is also distributed in the peripheral portion of the replacement fibrotic lesions. These findings suggest that tenascin is present in active fibrotic lesions and plays a role in the initiation of fibrotic changes that are observed in hearts with dilated cardiomyopathy.

Tenascin was found distributed in both the enlarged perimysium and endomysium of the fibrotic lesions, which are considered to have replaced the necrosed myocytes. Tenascin is an extracellular matrix glycoprotein consisting of six disulphide linked subunits with molecular masses of $190-250 \mathrm{kDa} .{ }^{9}$ The cDNA of human tenascin has been cloned and its amino acid sequence determined. ${ }^{10}$ Tenascin is expressed during development by several tissues. ${ }^{511-16}$ These biological studies indicate that tenascin promotes the early phase of ECM formation, especially through cell migration. The present results for the characteristic location of tenascin in dilated cardiomyopathy indicate that tenascin contributes to the fibrotic process in the early phase of initiation of cell migration in the fibrotic lesion, containing necrosed myocytes.

A recent study ${ }^{17}$ showed that in the wound healing process tenascin was first detected in the stroma later than fibronectin but disappeared earlier than fibronectin. Tenascin has been reported to be abundant in healing wounds but absent in old scars ${ }^{18}$; it is detected during the early phase of wound healing but is almost completely absent by the late stage. ${ }^{4}$ In the gastric mucosa, tenascin appears in the erosive mucosa but disappears after reepithelisation. ${ }^{6}$ Tenascin, with its chemoattractive effects, thus plays an important role in the initial wound healing process. This evidence indicates that the abundant tenascin staining observed in the extracellular matrix of the heart reflects active turnover, and that absent or diminished tenascin staining could indicate the presence of mature fibrotic lesions. The results of recent studies of tenascin and the present results indicate that fibrotic changes occur continuously in the heart with dilated cardiomyopathy.

Tenascin is detected in fetal skin wounds earlier than in adult skin wounds and the wound healing occurs more rapidly in the fetus. ${ }^{1920}$ Tenascin appeared before keratinocyte and fibroblast migration, and its early appearance in the fetal wounds, through its initiation of cell migration, may underlie the rapid epithelialisation seen in fetal wound healing. These studies generally indicate that fibroblasts infiltrate the lesions in which tenascin is abundant. In the biopsy specimens in the present study, staining for cell nucleus revealed that several fibroblasts were present at sites positively stained for tenascin. These results are consistent with reported findings.

In this study we could not clarify whether or not tenascin expression is specific for hearts with dilated cardiomyopathy. The tenascin expression was associated strongly with the presence of a replacement fibrotic lesion. This suggests that tenascin contributes to the fibrotic process of healing following myocyte damage. The same contribution to the healing process has been found in wound healing, ${ }^{4517}$ 
gastric mucosal injury, ${ }^{6}$ and liver injury. ${ }^{21}$ It is possible that tenascin may also be expressed in the fibrotic process following myocardial damage in other pathological conditions, such as ischaemic myocardial damage.

The small number of biopsy samples we examined is a limitation of this study, as is the failure to evaluate the relationship of tenascin staining to haemodynamic indices.

In summary, our results suggest that tenascin contributes to the fibrotic changes observed in the heart in dilated cardiomyopathy. Its variable distribution is thought to indicate that the phase of the fibrotic process differs from lesion to lesion.

1 Schwarz F, Mall G, Zobe H, Blickle J, Derks H, Manthey J, Wubler W. Quantitative morphologic findings of the myocardium in idiopathic dilated cardiomyopathy. $A m \mathcal{F}$ Cardiol 1983;51:501-6.

2 Chiquet M, Fambrough DM. Chick myotendinous antigen. 1. A monoclonal antibody as a marker for tendon and muscle morphogenesis. 2. A novel extracellular glycoprotein complex consisting of large disulfide-linked coprotein complex consisting of larg
subunits. $f$ Cell Biol 1984;98:1926-46.

3 Mackie EJ, Tucker RP, Halfter W, Chiquet-Ehrismann R The distribution of tenascin coincides with pathways of neural crest cell migration. Development 1988;102:

4 Mackie EJ, Halfter W, Liverani D. Induction of tenascin in healing wounds. F Cell Biol 1988;107:2757-67.

5 Murakami R, Yamaoka I, Sakakura T. Appearance of tenascin in healing skin of the mouse: possible involvement in seaming of wounded tissue. Int $\mathcal{f} \mathrm{Dev} B i o$ 1989;33:439-44.

6 Okada A. Expression of tenascin in human gastric mucosa. Mie Med F 1990;40:251-7.

$7 \mathrm{Hsu}$ SM, Raine L, Fanger H. Use of Avidin-BiotinPeroxidase Complex (ABC) in immunoperoxidase techniques. $¥$ Histochem Cytochem 1981;29:577-80.
8 Brandenburg RO, Chazov E, Falase AO, et al. Report of the WHO/ISFC task force on definition and classification of cardiomyopathies. Circulation 1981;64:437-8A

9 Oike $\mathrm{Y}$, Hiraiwa $\mathrm{H}$, Kawakatsu $\mathrm{H}$, et al. Isolation and characterization of human fibroblast tenascin. An extracellular matrix glycoprotein of interest for developmental studies. Int $\mathscr{\mathcal { F }}$ Dev Biol 1990;34:309-17.

10 Gulcher JR, Nies DE, Marton LS, Stefansson K. An alternatively spliced region of the human hexabrachion contains a repeat of potential N-glycosylation sites. Proc Natl Acad Sci USA 1989;86:1588-92.

11 Wehrle $B$, Chiquet $M$. Tenascin is accumulated along developing peripheral nerves and allows neurite outgrowth in vitro. Development 1990;110:401-15.

12 Aufderheide E, Chiquet-Ehrismann R, Ekblom P. Epithelial-mesenchymal interactions in the developing Epithelial-mesenchymal interactions in the developing
kidney lead to expression of tenascin in the mesenchyme. kidney lead to expression of ten

13 Chiquet-Ehrismann R, Mackie EJ, Pearson CA, Sakakura $T$. Tenascin: an extracellular matrix protein involved in tissue interactions during fetal development and oncogenesis. Cell 1986;47:131-9.

14 Mackie EJ, Thesleff I, Chiquet-Ehrismann R. Tenascin is associated with chondrogenic and osteogenic differentiation in vivo and promotes chondrogenesis in vitro. $\mathcal{F}$ Cell Biol 1987;105:2569-79.

15 Thesleff I, Mackie E, Vainio S, Chiquet-Ehrismann $R$. Changes in the distribution of tenascin during tooth development. Development 1987;101:289-96.

16 Vainio S, Jalkanen M, Thesleff I. Syndecan and tenascin expression is induced by epithelial-mesenchymal interactions in embryonic tooth mesenchyme. $\mathcal{F}$ Cell Biol tions in embryonic

17 Chuong CM, Chen HM. Enhanced expression of neural cell adhesion molecules and tenascin (cytotactin) during cell adhesion molecules and tenascin (cytotactin)
wound healing. Am $\mathcal{f}$ Pathol 1991;138:427-40.

18 Bourdon MA, Wikstrand CJ, Furthmayer H, Matthews TJ, Bigner DD. Human glioma-mesenchymal extracellular matrix antigen defined by monoclonal antibody. Cancer Res 1983;43:2796-805.

19 Whitby DJ, Longaker MT, Harrison MR, Adzick NS, Ferguson MW. Rapid epithelialization of fetal wounds is associated with the early deposition of tenascin. $\mathcal{F} \mathrm{Cell} \mathrm{Sci}$ 1991;99:583-6.

20 Whitby DJ, Ferguson MW. The extracellular matrix of lip wounds in fetal, neonatal and adult mice. Development 1991;112:651-68.

21 Yamada S, Ichida T, Matsuda $Y$, et al. Tenascin expression in human chronic liver disease and in hepatocellular carcinoma. Liver 1992;12:10-16. 